\title{
A Comprehensive Review on Classification of Oral Ulcerative Lesions
}

\author{
Hina Handa, ${ }^{*}$ Pooja Khare, ${ }^{2}$ Kriti Shrivastava ${ }^{3}$ \\ ${ }^{1,2}$ Department of Oral Medicine and Radiology, Peoples Dental Academy, Bhopal \\ ${ }^{3}$ Department of Oral Medicine and Radiology, Rishiraj Dental College, Bhopal
}

\begin{abstract}
A brief overview of the superficial oral lesion

Oral mucosal lesions are a broad group of lesions which are located in the soft tissues of the oral cavity identified by its specific etiology, clinical manifestations, differential diagnosis and treatment. White lesions of the oral cavity constitute a rather common group of lesions that are encountered during routine clinical dental practice. The process of clinical diagnosis and treatment planning is of great concern to the patient as it determines the prognosis. There should be in-depth knowledge to the consultant about the varied clinical features, etiology of the disease and various treatment plans. Clinical diagnostic skills and ideal judgment forms the key to successful management of white lesions of the oral cavity
\end{abstract}

This review will discuss many of the most common intraoral white lesions including their clinical presentation, how to make an accurate diagnosis

Keywords: Feeding, Nesting, Weaver-bird, Environmental conditions, Crops

\section{Introduction}

Oral lesions are a common clinical finding that we encounter day to day in clinics. Lesion may have varied features ranging from a small macular region to a white plaque or a patch.The lesion may have various forms of clinical features that include its colour, its presentation, flat or raised lesions and its different morphological forms. Surface of the lesion which may appear to be smooth, rough and corrugated surface. Diagnostic dilemma occurs to the practioners as the lesions presents with various specific clinical and morphological features. As a general practioners and interns we should be aware of the superficial oral lesions that may appear in the oral cavity and have sufficient knowledge and awareness to diagnose such lesions and if they appear suspected may send them for investigations. ${ }^{1}$

\section{Diagnostic Challenge}

A thorough patient history is important for diagnosis. For example, a history of heavy smoking, consumption of tobacco, arecanut etc.Possibility of a systemic condition should also be considered; for example, use of a medication known to cause a lichenoid reaction along with skin lesions suggests oral lichen planus. On examination, the location, and surface and sub epithelial characteristics of lesions are important factors in diagnosis. ${ }^{2}$

White lesions of the oral cavity (category wise in the decreasing order of frequency.

a. Inflammatory/Reactive

b. Dysplastic/neoplastic

\begin{tabular}{|c|c|}
\hline $\begin{array}{l}\text { Quick Response Code: } \\
\text { 口frig }\end{array}$ & $\begin{array}{l}\text { *Corresponding author: HinaHanda, Senior Lecturer, Mds-Oral Medicine Radiology, Peoples } \\
\text { Dental Academy Room No 5, Bhopal }\end{array}$ \\
\hline 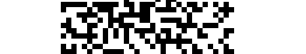 & Published: 10 December, 2020 \\
\hline 1. & $\begin{array}{l}\text { Citation: Hina H, Pooja K, Kriti S. A Comprehensive Review on Classification of Oral Ulcerative } \\
\text { Lesions. SOJ Den Oral Disor. 2020;1(1):1-5. DOI: 10.53902/SOJDOD.2020.01.000501 }\end{array}$ \\
\hline
\end{tabular}


c. Oral manifestations of common dermatologic disease (eg Lichen planus)
d. Oral manifestations of systemic disease
e. Developmental

The clinical examination to identify a lesion an oral condition and systematic approach to diagnose a lesion its specific characteristics may help in diagnosing an oral mucosal lesion. The examination of soft tissues in paediatricpatients involves knowledge of normal size, shape, colour, and texture of the structures that comprise it. The correct exploration of the oral mucosa can provide important tools in diagnosing developmental, neoplastic, infectious, or inflammatory alterations. ${ }^{3}$

Common superficial oral lesions include candidiasis; recurrent herpes labialise, recurrent aphthous stomatitis, erythema migrants, hairy tongue, and lichen planes. Diagnosing an oral lesion through its various clinical features and etiological factors may help

Table 1: Classical diagnostic features of oral superficial lesion. in evaluating and oral mucosal lesion. Knowledge of clinical characteristics such as size, location, surface morphology, colour, pain, and duration is helpful in establishing a diagnosis

Large-scale, population-based screening studies have identified the most common oral lesions as candidiasis, recurrent herpes labialise, recurrent aphthous stomatitis, mucocele, fibroma, mandibular and palatal tori, pyogenic granuloma, erythema migrans, hairy tongue, lichen planes, and leukoplakia. ${ }^{4}$

Surface debris that is easily wiped off suggests candidiasis, as it is scrap able mucosal lesion, while a speckled white patch that cannot be rubbed off is potentially precancerous, carcinoma in situ or squamous cell carcinoma. A homogeneous, striated white patch with no evidence of surface breach is likely to be benign. Lesions may have a warty surface.Verrucoid-papillary leukoplakia (verrucous hyperplasia), characterised by an irregular exophytic wart-like appearance, has been reported to be a diagnostic feature ${ }^{5}$ (Table 1 , Table 2, Table 3). ${ }^{6,7}$

\begin{tabular}{|c|c|}
\hline \multirow{10}{*}{ General description } & Yellow-pink to pearly white \\
\hline & Smooth surface \\
\hline & Soft to hard \\
\hline & Usually asymptomatic \\
\hline & Does not rub off \\
\hline & Submucosal thickening or enlargement \\
\hline & Plaque, popular or nodular pattern \\
\hline & Localized or multifocal distribution \\
\hline & Static, regresses or progresses \\
\hline & A cause may be present \\
\hline \multirow{3}{*}{ Focal flat } & Trauma \\
\hline & Cicatrix \\
\hline & Mucosal graft \\
\hline \multirow{8}{*}{ Focal, elevated } & Granular cell tumor \\
\hline & Lipoma \\
\hline & Oral-facial-digital syndrome \\
\hline & Epidermoid/dermoid cyst \\
\hline & Lymphoepithelial cyst \\
\hline & Soft tissue abscess \\
\hline & Parulis \\
\hline & Sialolith \\
\hline \multirow{9}{*}{ Diffuse or multifocal, flat } & Trauma \\
\hline & Cicatrix \\
\hline & Child abuse \\
\hline & Resolved aphthous major \\
\hline & Factitial injury \\
\hline & Lesch-Nyhan syndrome \\
\hline & Hereditary sensory syndromes with oral mutilation \\
\hline & Seizure disorder \\
\hline & Hairy tongue \\
\hline
\end{tabular}


Diffuse or multifocal, elevated

\begin{tabular}{|c|}
\hline Gingival cysts of the newborn \\
\hline Palatal cysts of the new-born \\
\hline Lymphoid aggregates \\
\hline Fordyce granules6 \\
\hline
\end{tabular}

Table 2: Common superficial oral lesions.

\begin{tabular}{|c|c|}
\hline Condition & Clinical features \\
\hline \multirow{3}{*}{ Candidiasis } & Pseudomembranous: adherent white plaques that may be wiped off \\
\hline & Erythematous:red macular lesions, often with a burning sensation \\
\hline & Perleche (angular cheilitis):erythematous, scaling fissures at the corners of the mouth \\
\hline Recurrent Herpes labialis & $\begin{array}{c}\text { Prodrome (itching, burning, tingling) lasts approximately } 12 \text { to } 36 \text { hours, followed by eruption of clustered vesicles } \\
\text { along the vermilion border that subsequently rupture, ulcerate, and crust }\end{array}$ \\
\hline Recurrent aphthous stomatitis & Ulcers surfaced by a yellowish-white pseudo membrane surrounded by erythematous halo \\
\hline Erythema migrans & Migrating lesions with central erythema surrounded by white-to-yellow elevated borders; typically on tongue \\
\hline \multirow{2}{*}{ Lichen planus } & Reticular:white, lacy striae \\
\hline & Erosive:erythema and ulcers with peripheral radiating striae, erythematous and ulcerated gingival.7 \\
\hline
\end{tabular}

Table 3: Systemic approach for diagnosing oral lesion.

\begin{tabular}{|c|}
\hline History of current illness-Onset, Duration, frequency, intensity Aggravating and relieving factors \\
\hline Medical history-Systemic history causing oral lesions \\
\hline Habit history-Alcohol,tobacco, smoking \\
\hline Drug history \\
\hline Clinical examination-intraoral, lesion inspection adjunctive visual tool such as toludine blue,vizilite \\
\hline Differential diagnosis \\
\hline Investigation-biopsy \\
\hline Final diagnosis \\
\hline Management of lesion \\
\hline Habit counseling \\
\hline
\end{tabular}

\section{Brief Description}

\section{Candidiasis}

Candidiasis is the most common oral fungal infection caused by candida albicans. The pseudomembranous and hyperplastic type of candidiasis present as white lesions of the oral cavity. Pseudomembranous candidiasis has white plaques resembling curdled milk Scraping the white lesion can remove the plaques. The hyperplastic candidiasis or candidal leukoplakia presents as non-scrapable white patch usually located on the anterior buccal mucosa. The diagnosis is confirmed by the presence of candidal hyphae in the lesions. There is resolution of the lesion after antifungal therapy.

\section{Lichen Planus}

Lichen planus is an inflammatory dermatologic disease frequently manifesting in the oral cavity and usually involves some degree of hyperkeratosis or epithelial hyperplasia. The condition usually presents as a white lesion with clear cut and distinguishable features enabling the dental surgeon to make a reliable diagnosis based on clinical features alone.

The most common presentation of Oral lichen planus is as follows

a. Somewhat common in adults b. Women are more affected than men

c. Keratotic plaques or white striae are bilaterally distributed, buccal mucosa being a common site.

d. Lesions are painless unless they are of the erosive variety.

e. Surrounding oral mucosa may appear erythematous with a dusky red discoloration.

f. Lesions show remissions and exacerbations which are said to be associated with periods of stress. ${ }^{9}$

\section{Herpes Labialis}

Primary oral infection with the herpes simplex virus (HSV) typically occurs at a young age, is asymptomatic, and is not associated with significant morbidity. A minority of persons develop a symptomatic primary infection, presenting with an acute outbreak of oral vesicles that rapidly collapse to form zones of erythema and ulceration. In all cases, the gingiva is involved; in addition, other oral mucosal sites and the perioral skin may be affected.They classically manifest as a well-localized cluster of small vesicles along the vermilion border of the lip or adjacent skin The vesicles subsequently rupture, ulcerate, and crust within 24 to 48 hours. Spontaneous healing occurs over seven to 10 days. ${ }^{10}$ 


\section{Ras-Recurrent Aphthous Stomatitis}

Recurrent aphthous stomatitis is characterized by recurring, painful, solitary or multiple ulcers, typically covered by a whiteto-yellow pseudomembrane and surrounded by an erythematous halo (Figure 1). Recurrent aphthous stomatitis usually involves nonkeratinizing mucosa (e.g., labial mucosa, buccal mucosa, ventral tongue). There are three clinical forms: minor, major, and herpetiform. The minor form is the most common and appears as rounded, well-demarcated, single or multiple ulcers less than $1 \mathrm{~cm}$ in diameter that usually heal in 10 to 14 days without scarring. The underlying etiology is not clear, though a series of factors are known to predispose to the appearance of oral aphthae, including genetic factors, food allergens, local trauma, endocrine alterations (menstrual cycle), stress and anxiety, smoking cessation, certain chemical products and microbial agents. Although a variety of host and environmental factors have been implicated, the precise pathogenesis remains unknown. Smoking is associated with a lower prevalence, but other associations, such as nutritional deficiencies (e.g., vitamin B12, folate, iron), remain unclear.33Severe cases may be related to underlying systemic conditions such as inflammatory bowel disease, celiac disease, Behçet's syndrome, and HIV infection. ${ }^{11}$ Three clinical subtypes of RAS have been established according to the magnitude, number and duration of the outbreaks.

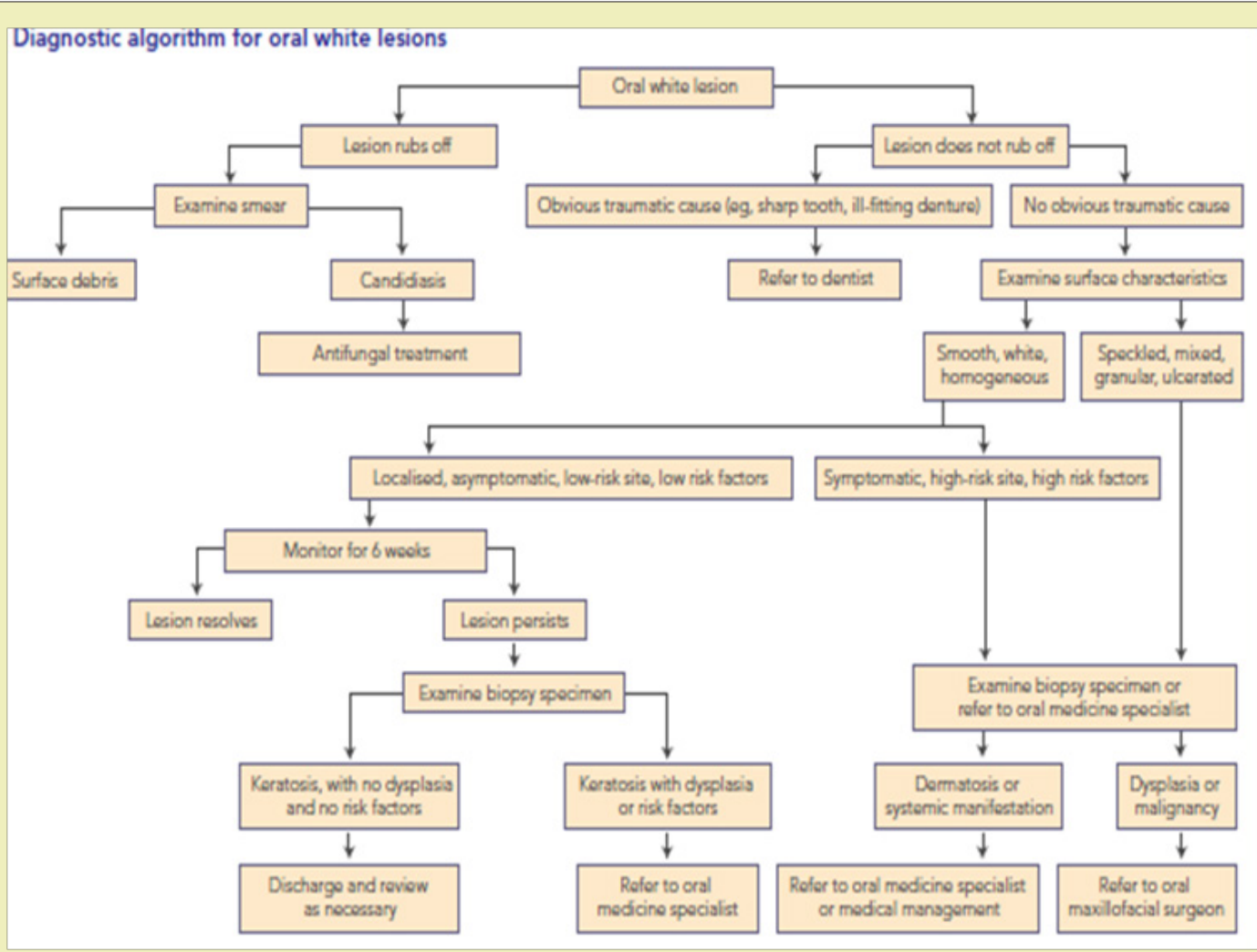

Figure 1: Diagnosing oral mucosal lesions.

Minor RAS: This is the most common presentation of the disease, representing $70-85 \%$ of all cases. It manifests as small rounded or oval lesions covered by a grayish-white pseudomembrane and surrounded by an erythematous halo. Each minor RAS episode usually involves the appearance of 1-5ulcers measuring under $1 \mathrm{~cm}$ in diameter. These episodes are self-limiting and resolve within 4-14days without leaving scars. Major RAS: This is the most severe presentation of the disease, representing $10 \%$ of all cases. In this subtype the ulcers measure over $1 \mathrm{~cm}$ in size and tend to appear on the lips, soft palate and pharynx. The lesions persist for over 6weeks and can leave scars.

Herpetiform RAS: This subtype accounts for $1-10 \%$ of all cases and is characterized by recurrent outbreaks of small, deep and painful ulcers. Up to 100 aphthae can develop simultaneously, measuring $2-3 \mathrm{~mm}$ in size, though they tend to merge to form larger ulcerations with an irregular contour. The diagnosis is based on a detailed clinical history, together with complementary procedures such as laboratory tests, where required. The predisposing factors must be identified and controlled. The severity of the outbreak must be evaluated, along with the type of aphthae and the frequency of outbreaks. Only when the outbreaks and symptoms are continuous should drug treatment be used, and starting in all cases with local drug treatment. ${ }^{12}$

\section{Erythema Migrans}

Erythema migrans may occur in children and adults and exhibits a female predilection. Tongue lesions exhibit central erythema caused by atrophy of the filiform papillae and usually is surrounded by slightly elevated, curving, white-to-yellow borders. ${ }^{13}$ Erythema migrans typically involves the dorsum of the tongue, sometimes the ventrum, and rarely other areas on the oral mucosa. There are ir- 
regular, pink or red depapillated map-like areas, which change in shape, increase in size, and spread or move to other areas, sometimes within hours the red areas are often surrounded by distinct yellowish, slightly raised margins.There is increased thickness of the intervening filiform papillae. It is mostly asymptomatic.

A small minority complain of soreness and these patients are virtually invariably middle-aged. If sore, this may be noted especially with acidic foods (e.g. tomatoes, strawberries, pineapple, walnuts or some cheese). Why the condition should give rise to symptoms after it has presumably been present for decades is unclear (Figure 1). ${ }^{13}$

\section{Hairy Tongue}

Hairy tongue is an acquired benign pathology. Clinically, is characterized by hair like projections, which, are due to the presence of elongated and hypertrophied filiform papillae. ${ }^{14}$ It usually occurs in the two-thirds anterior to the circumvallated papillae on the dorsal tongue surfacethe diagnosis of hairy tongue is clinical. Patients generally report smoking and poor oral hygiene presence of plaques whose colour may vary from white, tan, bluish, yellowish, green brown or black in the anterior two thirds of the tongue. An important feature is that the area of the lesion can be scraped off. The lesion is usually asymptomatic; however, the patient may report complaints of metallic taste, dysgeusia, nausea, burning mouth and halitosis however, the use of antibiotics (penicillin, aureomycin, erythromycin, doxycycline and neomycin), candidiasis, alcohol, and dentures are associated with this pathology. ${ }^{15}$

Oral mucosal lesions may occasionally diagnosed correctly, based on a brief history and clinical examination.1 therefore, an accurate description is essential for diagnosis and management of oral lesions. As regards, continuous observation and monitoring of the lesion are required, an acceptable description would be critical for comparison purposes. When biopsy sample is taken, the pathologist needs an accompanying lesion description to reach a definitive diagnosis. Since, questions about the management of lesion may arise, documentation of the description is necessary and should be included in patient records. ${ }^{2}$ Lack of detailed description of oral lesions, can lead to difficulties in prioritizing the oral medicine appointments. Oral mucosal lesions may occasionally diagnose correctly, based on a brief history and clinical examination. ${ }^{1}$ therefore, an accurate description is essential for diagnosis and management of oral lesions. As regards, continuous observation and monitoring of the lesion arerequired, an acceptable description would be critical for comparison purposes. When biopsy sample is taken, the pathologist needs an accompanying lesion description to reach a definitive diagnosis. Since, questions about the management of lesion may arise, documentation of the description is necessary and should be included in patient records. ${ }^{2}$ Lack of detailed description of oral lesions, can lead to difficulties in prioritizing the oral medicine appointments.

Superficial oral lesions of the oral cavity are commonly encountered during clinical dental practice. Although different lesions have different characteristic features, benign physiologic entities may present as white lesions, systemic conditions, infections, and malignancies may also present as white or red oral lesions. A thorough chair side investigation is considered important for early diagnosis and efficient treatment of a particular disease, whether; a particular white lesion can turn problematic in future. It is required to categorize the lesion and determine whether it belongs to the commonly seen reactive group or to the more dangerous precancerous group.

\section{Conclusion}

There are certain superficial lesions which after evaluation too have clinical diagnostic dilemma. The process of clinical diagnosis of white lesion which is based on various appearances which may prove to be challenge for the clinician. Early diagnosis of the lesion and tracing the etiological factors and treating the patient timely may prevent the widespread of the disease; likewise removal of a chronic irritant which can lead to traumatic oral ulcerative lesions should be treated on time to prevent the aggressiveness of the lesion. While most intraoral white lesions are benign, some are premalignant and/or malignant at the time of clinical presentation, making it extremely important to accurately identify and appropriately manage these lesions. Due to their similar clinical appearances, it may be difficult sometimes to differentiate benign white lesions from their premalignant/malignant counterparts.

\section{References}

1. Paul JR, Epstein JB. White lesions of the oral cavity-An aid to accurate diagnosis. Oral health. 1980;70(12):51-74.

2. White Lesions of the Oral Mucosa-A Diagnostic Dilemma Soukos N. Oral leukoplakia, idiopathic. Emedicine. Omaha, Neb: Medscape, 2008.

3. Espinoza I, Rojas, R, Aranda W, Gamonal J. Prevalence of oral mucosal lesions in elderly people in Santiago, Chile. J Oral Pathol Med. 2003;32(10):571-575.

4. Shulman JD, Beach MM, Rivera Hidalgo F. The prevalence of oral mucosal lesions in U.S. adults: data from the Third National Health and Nutrition Examination Survey, 1988-1994. J Am Dent Assoc. 2004;135(9):12791286

5. Silverman S, Gorsky M, Lozada F. Oral leukoplakia and malignant transformation: a follow-up study of 257 patients. Cancer. 1984;53(3):563-568.

6. Davis Massey. Potential pitfalls in diagnostic oral pathology: a review for the general surgical pathologist Adv Anat Pathol. 2005;12(6):332-349.

7. Chan ES, Thornhill M, Zakrzewska J. Interventions for treating oral lichen planus. Cochrane Database Syst Rev. 2000;(2).

8. Wood NK, Goaz PW. Differential Diagnosis of Oral and Maxillofacial Lesions. $5^{\text {th }}$ edition; 1997: Elsevier.

9. Do Prado RF, Marocchio LS, Felipini RL. Oral Lichen Planus versus Oral lichenoid reaction; Difficulties in diagnosis. Ind J Dent Res. 2009; 20(3):361-364.

10. Neville BW. Herpes simplex virus. In: Oral and Maxillofacial Pathology. Philadelphia, Pa: Saunders, 2002:213-220.

11. Ogura M, Yamamoto T, Morita M, et al. A case control study on food intake of patients with recurrent aphthous stomatitis. Oral Surg Oral Med Oral Pathol Oral Radiol Endod. 2001;91(1):45-49.

12. Zhou Y, Chen Q Meng W, et al. Evaluation of penicillin G potassium troches in the treatment of minor recurrent aphthous ulceration in 
a Chinese cohort:a randomized, double-blinded, placebo and notreatment-controlled, multicenter clinical trial. Oral Surg Oral Med Oral Pathol Oral Radiol Endod. 2010;109(4):561-566.

13. Assimakopoulos D, Patrikakos G, Fotika C, et al. Benign migratory glossitis or geographic tongue: an enigmatic oral lesion. Am J Med. 2002;113(9):751-755.
14. Villa A, Woo S Bin. Leukoplakia-A Diagnostic and Management Algorithm. J Oral Maxillofac Surg. 2017;75(4):723-734.

15. Gurvits GE, Tan A. Black hairy tongue syndrome. World J Gastroenterol. 2014;20(31):10845-10850. 